\title{
LIOUVILLE TYPE THEOREMS FOR MAPPINGS WITH BOUNDED (CO)-DISTORTION
}

\author{
by M. TROYANOV \& S. VODOP'YANOV*
}

\section{Introduction.}

A mapping $f: M \rightarrow N$ between oriented $n$-dimensional Riemannian manifolds is said to have bounded $s$-distortion (or $s$-dilatation) $(1 \leqslant s<\infty)$ if $f \in W_{\mathrm{loc}}^{1,1}(M, N)$ and

$$
\left|d f_{x}\right|^{s} \leqslant K J_{f}(x)
$$

a.e. $x \in M$.

The Sobolev class of mappings $W_{\text {loc }}^{1,1}(M, N)$ is defined in Section 3 below; these mappings have a formal differential $d f_{x}: T_{x} M \rightarrow T_{f(x)} N$ almost everywhere; in the above inequality, $\left|d f_{x}\right|$ denotes its operator norm and $J_{f}(x)=\operatorname{det} d f_{x}$ its Jacobian.

Mappings with bounded $s$-distortion are generalizations of quasiregular mappings; they have been studied (under various names and viewpoints) since about 30 years, see [6], [8], [24], [25], [28], [30], [41], [44] among other works. In the special case of homeomorphisms with bounded $s$-distortion with $s>n-1$, a metric characterization has been given in [8].

These mappings originated as suitable class of mappings in the change-of-variable formula for functions in the Sobolev spaces $\mathcal{L}^{1, s}$ (see Section 4). As it turns out, this class of mappings feels quite well the

* This work is partially supported by the INTAS, grant $\mathrm{N}^{\mathrm{O}} 10170$.

Keywords: Mapping with bounded distortion - Capacity - Parabolicity.

Math. classification: 30C65 - 31B15 - 26B10. 
asymptotic geometry of Riemannian manifolds. In [6], J. Ferrand was able to prove that a Riemannian manifold is characterized up to bilipschitz equivalence by its Royden algebra; the proof heavily uses the theory of mappings with bounded s-distortion. In [28], P. Pansu gave conditions on the geometry of manifolds implying that mappings with bounded $s$ distortion are quasi-isometries. The work of Ferrand and Pansu has been extended to the case of metric measure spaces in the recent thesis of K. Gafaïti.

Mapping with bounded $s$-distortion are a subclass of the so-called mappings with finite distortion which are defined by the condition that $J_{f} \in L_{\mathrm{loc}}^{1}$ and $\left|d f_{x}\right|^{n} \leqslant \Phi(x) J_{f}(x)$ where $\Phi(x)$ is finite a.e. (see the argument in the proof of Corollary 7.1). Mappings with finite distortion play an important role in non-linear elasticity (see e.g. [27]) and they are now being intensively studied. See e.g. the papers [16], [18], [41] and the rich references therein.

Another important generalization of mappings with bounded $s$ distortion is given by the class of mappings such that $\left|\Lambda_{k} f_{x}\right|^{q} \leqslant K J_{f}(x)$ where $\Lambda_{k} f$ is the $k$-th. exterior power of $d f$, i.e. the effect of $d f_{x}$ at the level of $k$-forms. These mappings appear in $L^{p}$ cohomology; see e.g. the recent paper [29] of P. Pansu, where flows of such mappings are used in the computation of $L^{p}$-cohomology of manifolds with negative curvature and solvable Lie groups.

In the present paper, we will consider the case $k=n-1$; let us thus define a mappings with bounded q-codistortion $(1<q<\infty)$ to be a mapping $f \in W_{\text {loc }}^{1,1}(M, N)$ for which there exists a constant $K^{\prime}$ such that

$$
\left|\Lambda_{n-1} f_{x}\right|^{q} \leqslant K^{\prime} J_{f}(x) \quad \text { a.e. }
$$

We now state a number of questions, concerning mappings with bounded $s$-distortion, we are interested in

1) What are the obstructions to the existence of a non constant mapping with bounded $s$-distortion $f: M \rightarrow N$ ?

2) Describe the set of all $s \geqslant 1$ for which there exists a homeomorphism (or a diffeomorphism) $f: M \rightarrow N$ with bounded $s$-distortion.

3) Suppose that $f: M \rightarrow N$ is a non constant mapping with bounded $s$-distortion: How big may the omitted set $N \backslash f(M)$ be? (In particular, when can it be said that $f$ is onto?)

4) Assuming that $f: M \rightarrow N$ is a continuous mapping with bounded 
$s$-distortion. What can be said about the topological and or the geometrical properties of $f$ ?

Similar questions may be raised about mappings with bounded $q$ codistortion.

We will give some answers to all of these questions. The main techniques we use are based on potential theory: Recall that a condenser in $M$ is a pair $(D, A)$ where $A \subset M$ is a connected open subset and $D \subset A$ is compact. The $p$-capacity of the condenser $(D, A)$ is defined as

$$
\operatorname{Cap}_{p}(D, A)=\inf \left\{\int_{A}|\nabla u|^{p}: u \in C_{0}^{0}(A) \cap W^{1, p}(A) \text { and } u \geqslant 1 \text { on } D\right\} .
$$

Definition. - The manifold $M$ is $p$-parabolic if $\operatorname{Cap}_{p}(D, M)=0$ for all compact subsets $D \subset M$ and $p$-hyperbolic otherwise.

We have included in Section 7.2 below a brief discussion of this notion.

An answer to the first question above is the following Liouville type theorem:

Theorem. - Let $M$ and $N$ be oriented $n$-dimensional Riemannian manifolds and let $f \in W_{\mathrm{loc}}^{1, n}(M, N)$ be a mapping with bounded s-distortion with $s>(n-1)$. Assume that $M$ is $p$-parabolic, where $\frac{1}{p}+\frac{n-1}{s}=1$. Then either $f$ is constant a.e. or $N$ is also p-parabolic.

This result is a consequence of Theorem A and Corollary 7.1 in the present paper; it is in fact proved for a wider class than $W_{\text {loc }}^{1, n}(M, N)$.

In the special case of quasi-regular mappings (i.e. $s=p=n$ ), this result has been obtained around 1968 by Y. Reshetnyak and, independently, by O. Martio, S. Rickman and J. Väisälä (see [32] and [22]). At the end of the paper we shortly recall the original argument of Reshetnyack.

Some answers to the other questions mentioned above are given in Sections 2, 4 and 7 .

The paper is organized as follow: In Section 2, we give some additional definitions, state the main results of the paper and give some corollaries. In Section 3 we recall some basic facts about Sobolev mappings, in Section 4 we discuss homeomorphisms with bounded $s$-distortion and in Section 5 we prove a capacity inequality. After these preparations, we prove the main theorems in Section 6. Finally, in Section 7, we give some complementary information on mappings with bounded $s$-distortion. 


\section{Definitions and statement of the results.}

Throughout the paper $M$ and $N$ are oriented, connected $n$-dimensional Riemannian manifolds. We denote by $d \mu$ and $d \nu$ the volume elements of $M$ and $N$ respectively.

In order to state our results, we need some additional definitions:

Definitions. - (1) The map $f$ has essentially finite multiplicity if $\mathrm{N}_{f}(M)<\infty$, where

$$
\mathrm{N}_{f}(A):=\operatorname{ess}_{\sup _{y}} \operatorname{Card}\left(f^{-1}(y) \cap A\right)
$$

for any measurable subset $A \subset M$.

(2) A continuous map is open and discrete if the image of any open set $U \subset M$ is an open set $f(U) \subset N$ and the inverse image $f^{-1}(y)$ of any point $y \in N$ is a discrete subset of $M$. The branch set of such a mapping is the set $B_{f} \subset M$ of points $x \in M$ such that $f$ is not a local homeomorphism in a neighborhood of $x$.

The next two definitions are regularity assumptions. They are always satisfied if one assumes e.g. that $f$ is locally Lipschitz, or that $f \in$ $W_{\text {loc }}^{1, s}(M, N)$ for $s>n$, or that $f$ is locally quasi-regular.

(3) A measurable map $f: M \rightarrow N$ satisfies Lusin's property if the image of any set $E \subset M$ of measure zero is a set $f(E) \subset N$ of measure zero.

An important and well-known result (see Proposition 3.2) states that for any map $f: M \rightarrow N$ belonging to $W_{\text {loc }}^{1,1}(M, N)$ there exists a sequence of compact sets $A_{i} \subset M$ such that the restriction of $f$ to each $A_{i}$ is Lipschitz and the complementary set $E_{f}:=M \backslash \cup_{i} A_{i}$ has measure zero. We call $E_{f}$ the exceptional set of $f$.

(4) The map $f \in W_{\text {loc }}^{1,1}(M, N)$ is almost absolutely continuous if it is continuous and for any bounded domain $\Omega \Subset M$ the following property holds: for any $\varepsilon>0$ we can find $\delta=\delta(\Omega, \varepsilon)>0$ such that for any finite or infinite sequence of pairwise disjoint balls $\left\{B\left(x_{i}, r_{i}\right)\right\}$ contained in $\Omega$ with center $x_{i} \in E_{f}$, we have

$$
\sum \operatorname{vol}\left(B_{i}\right) \leqslant \delta \quad \Longrightarrow \quad \sum \operatorname{diam}\left(f B_{i}\right)^{n}<\varepsilon
$$

Remark. - The notion of almost absolute continuity appeared in [41], [42]; it is a generalization of absolute continuity in the sense of Malý 
as defined in [19]. In particular any mapping in $W_{\text {loc }}^{1, p}(M, N)$ with $p>n$ and any continuous mapping in $W_{\text {loc }}^{1, n}\left(\mathbb{R}^{n}, \mathbb{R}^{n}\right)$ with monotone coordinate functions is an example of almost absolutely continuous mapping, see [19].

In dimension 2, a mapping has bounded $s$-distortion if and only if it has bounded $s$-codistortion. In higher dimension, we have the following relation between distortion and codistortion:

LEMma 2.1. - Let $f: M \rightarrow N$ be a mapping with bounded $s$ distortion for some $s>n-1$, then $f$ has bounded q-codistortion for $q=s /(n-1)$.

Conversely, if $f: M \rightarrow N$ is a mapping with bounded q-codistortion for some $q<\frac{n-1}{n-2}$ such that $J_{f}>0$ a.e., then $f$ has bounded $s$-distortion for $s=\frac{q}{(n-1)-q(n-2)}$.

The exponents in this lemma are sharp.

Proof. - It is a trivial consequence of the inequalities

$$
\left|\Lambda_{n-1} f_{x}\right| \leqslant\left|d f_{x}\right|^{n-1} \quad \text { and } \quad\left|d f_{x}\right| J_{f}(x)^{n-2} \leqslant\left|\Lambda_{n-1} f_{x}\right|^{n-1} .
$$

We now state the main results of the present paper:

Theorem A. - Let $f \in W_{\mathrm{loc}}^{1, s}(M, N)$ be a continuous open and discrete mapping with bounded $s$-distortion, where $s>(n-1)$, satisfying Lusin's property. If $M$ is $p$-parabolic with $p=\frac{s}{s-(n-1)}$, then $N$ is also p-parabolic.

Recall that a map $f \in W_{\text {loc }}^{1, s}$ always satisfies Lusin's property if $s>n$. In Section 3 below we give other sufficient conditions. In Section 7.1 below, we will also give sufficient conditions for a continuous mapping with bounded $s$-distortion to be discrete and open.

The next result is an analog of Theorem A. It holds without any topological restrictions but assumes that $f$ has finite essential multiplicity:

Theorem B. - Let $f \in W_{\mathrm{loc}}^{1, s}(M, N)$ be a mapping of essentially finite multiplicity with bounded $s$-distortion where $s>(n-1)$. Assume either

1) $\left|\Lambda_{n-1} f\right| \in L_{\mathrm{loc}}^{n /(n-1)}(M)$, or

2) $f$ is almost absolutely continuous and $J_{f} \in L_{\mathrm{loc}}^{1}(M)$. 
If $M$ is $p$-parabolic with $p=\frac{s}{s-(n-1)}$, then either $f$ is constant a.e. or $N$ is also p-parabolic.

In Theorem B (under assumption 2) no continuity is assumed. The proofs of theorems $\mathrm{A}$ and $\mathrm{B}$ are based on quite different approaches; it would be interesting to have a unified method proving both results.

Remark 1. - These results are sharp. They say for instance that there is no mapping of finite essential multiplicity with bounded $s$-distortion from the Euclidean space to the hyperbolic space for $s>(n-1)$. This is optimal since the Riemannian exponential exp : $T_{x_{0}} \mathbb{H}^{n} \rightarrow \mathbb{H}^{n}$ (where $\mathbb{H}^{n}$ is the hyperbolic space) is a diffeomorphism with bounded $(n-1)$-distortion. Other comments on the optimality of these results are given in [7].

Theorem B will be obtained as a consequence of the following result on mappings with bounded codistortion:

Theorem C. - Let $f: M \rightarrow N$ be a mapping of essentially finite multiplicity with bounded $q$-codistortion where $q>1$. Suppose that $J_{f}>0$ on some set of positive volume. Assume furthermore either

1) $f \in W_{\mathrm{loc}}^{1, n-1}(M, N)$ and $\left|\Lambda_{n-1} f\right| \in L_{\mathrm{loc}}^{n /(n-1)}(M)$, or

2) $f$ is almost absolutely continuous, $f \in W_{\mathrm{loc}}^{1, s}(M, N)$ for some $s>(n-1)$ and $J_{f} \in L_{\text {loc }}^{1}(M)$.

If $M$ is $p$-parabolic with $p=q /(q-1)$, then $N$ is also $p$-parabolic.

Remark 2. - The condition that $J_{f}>0$ on some set of positive volume cannot be replaced by the weaker condition that $f$ is not constant a.e. For instance, look at the hyperbolic three-space in the upper-half space model $\mathbb{H}^{3}=\left\{(x, y, z) \in \mathbb{R}^{3} \mid z>0\right\}$ (with metric tensor $d s^{2}=$ $\left.\left.\left(d x^{2}+d y^{2}+d z^{2}\right) / z^{2}\right)\right)$. Then the mapping $f: \mathbb{R}^{3} \rightarrow \mathbb{H}^{3}$ given by $f(x, y, z)=(x, 0,1)$ is of finite essential multiplicity and has bounded $q$ codistortion for all $q \geqslant 1$. Yet $\mathbb{H}^{3}$ is $p$-hyperbolic for all $p$ and $\mathbb{R}^{3}$ is $p$-parabolic for all $p \geqslant 3$.

The next result goes in the other direction:

Theorem D. - Let $f \in W_{\text {loc }}^{1,1}(M, N)$ be a continuous non constant proper mapping with bounded s-distortion of finite essential multiplicity. If $M$ is s-hyperbolic, then so is $N$.

Remark 3. - The hypothesis that $f$ is proper is necessary. For 
instance if $N$ is a compact manifold and $M \subset N$ is an open domain whose complement $N \backslash M$ has non empty interior, then $N$ is $s$-parabolic for all $s$ and $M$ is $s$-hyperbolic for all $s \in[1, \infty]$. Yet the inclusion $f: M \hookrightarrow N$ has bounded $s$-distortion for all $s$.

We now give some applications of our results. We begin by a Picard type theorem for mappings with bounded $s$-distortion.

Corollary 2.1. - Let $f: M \rightarrow N$ be a continuous mapping with bounded $s$-distortion, $s>(n-1)$ satisfying the hypothesis of Theorem $A$. Assume that the manifold $M$ is $p$-parabolic where $p:=\frac{s}{s-(n-1)}$. Then $f$ is surjective if $p>n$, and the omitted set $N \backslash f(M)$ has Hausdorff dimension $\leqslant(n-p)$ if $p \leqslant n$.

Proof. - Observe that $f$ actually maps $M$ onto $N^{\prime}=f(M)$ (which is an open subset of $N$ ). By Theorem A, the manifold $N^{\prime}$ is thus $p$-parabolic and therefore the Hausdorff dimension of $N \backslash N^{\prime}$ is $\leqslant n-p$.

For a quasiregular mapping on Euclidean space $f: \mathbb{R}^{n} \rightarrow \mathbb{R}^{n}$, a stronger result is due to S. Rickman. He proved that $f$ omits at most finitely many points (see theorem 2.1 in [34], chapter IV]).

Corollary 2.2. - Let $f: M \rightarrow N$ be an injective $C^{1}$ mapping with bounded $q$-codistortion. Assume that $q<\frac{n}{n-1}$ and that $M$ is $p$ parabolic with $p=\frac{q}{q-1}$. Then $f$ is a diffeomorphism.

For the proof of this corollary, will need a lemma. Recall that the principal dilatation coefficients (or singular values) at $x \in M$ of a mapping $f \in W_{\text {loc }}^{1,1}(M, N)$ are the square roots $\lambda_{1} \leqslant \lambda_{2} \leqslant \ldots \leqslant \lambda_{n}$ of the eigenvalues of $d f_{x} d f_{x}^{t}$; they are defined almost everywhere. Observe the following useful inequalities:

$$
\left|d f_{x}\right|=\lambda_{n}, \quad\left|J_{f}(x)\right|=\lambda_{1} \cdot \lambda_{2} \cdots \lambda_{n}, \quad\left|\Lambda_{n-1} f_{x}\right|=\lambda_{2} \cdot \lambda_{3} \cdots \lambda_{n} .
$$

Lemma 2.2. - Let $f: M \rightarrow N$ be a mapping with bounded $q$-codistortion. If $q<\frac{n}{n-1}$ then either $J_{f}=0$ a.e. or there exists a constant $\delta>0$ such that all the principal dilatation coefficients are almost everywhere $\geqslant \delta$.

Proof. - Let $\lambda_{1} \leqslant \lambda_{2} \leqslant \ldots \leqslant \lambda_{n}$ be the dilatation coefficients of $f$ at $x$. We have by hypothesis $\left|\Lambda_{n-1} f_{x}\right|^{q} \leqslant K J_{f}(x)$ a.e., i.e. $\left(\lambda_{2} \cdot \lambda_{3} \cdots \lambda_{n}\right)^{q} \leqslant$ 
$K\left(\lambda_{1} \cdot \lambda_{2} \cdots \lambda_{n}\right)$. This implies $\lambda_{1}^{(n-1)(q-1)} \leqslant\left(\lambda_{2} \cdot \lambda_{3} \cdots \lambda_{n}\right)^{q-1} \leqslant K \lambda_{1}$, from which one obtains $\lambda_{1} \geqslant K^{1 /(n+q-n q)}$, provided $q<\frac{n}{n-1}$.

Proof of Corollary 2.2. - By the previous lemma, all principal dilatation coefficients are bounded below, in particular $f$ is a local diffeomorphism. Assume now that $f$ is not surjective. Then there exists a point $y_{0} \in N \backslash f(M)$. Let $N^{\prime}:=N \backslash\left\{y_{0}\right\}$, this is a $p$-hyperbolic manifold (since $p>n)$. By Theorem $\mathrm{C}$, the manifold $M$ must therefore be $p$-hyperbolic; but this contradicts the hypothesis and we thus conclude that $f$ is surjective.

If $M=N=\mathbb{R}^{n}$, we don't need to assume global injectivity in the previous corollary.

Corollary 2.3. - Let $f: \mathbb{R}^{n} \rightarrow \mathbb{R}^{n}$ be a $C^{1}$ mapping with bounded $q$-codistortion where $q<\frac{n}{n-1}$ and such that $J_{f} \not \equiv 0$. Then $f$ is a global diffeomorphism.

Proof. - By Lemma 2.2 all the eigenvalues of $d f_{x}^{t} d f_{t}$ are uniformly bounded below. We thus conclude from a recent theorem of M. Chamberland and G. Meister that $f$ is injective (see [1], th. 1.1).

Now set $p:=\frac{q}{q-1}$, then $p>n$ and hence $\mathbb{R}^{n}$ is $p$-parabolic. We conclude the proof from the previous corollary.

We also have similar results for mappings with bounded $s$-distortion.

Corollary 2.4. - Let $f: M \rightarrow N$ be an injective $C^{1}$ mapping with bounded $s$-distortion.

Assume that $(n-1)<s<n$ and that $M$ is $p$-parabolic with $p=\frac{s}{s-(n-1)}$. Then $f$ is a diffeomorphism.

The proof is similar to that of Corollary 2.2.

Corollary 2.5. - Let $f: \mathbb{R}^{n} \rightarrow \mathbb{R}^{n}$ be a non constant $C^{1}$ mapping with bounded s-distortion where $(n-1)<s<n$. Then $f$ is a global diffeomorphism.

Proof. - This is clear from Lemma 2.1 and the previous corollaries.

This last result also holds for $s=n \geqslant 3$. Indeed, V.A. Zorich has 
proved that a quasi-regular mapping $f: \mathbb{R}^{n} \rightarrow \mathbb{R}^{n}$, which is a local homeomorphism is in fact a global homeomorphism provided $n \geqslant 3$, see $[46]$.

\section{Calculus of Sobolev mappings.}

Since a mapping $f: M \rightarrow \mathbb{R}^{m}$ is given by its components which are $n$ functions: $f=\left(f_{1}, f_{2}, \ldots, f_{m}\right)$, it is natural to say that $f$ belongs to the Sobolev space $W_{\text {loc }}^{1, s}\left(M, \mathbb{R}^{m}\right)$ if each component $f_{i} \in W_{\text {loc }}^{1, s}(M, \mathbb{R})$.

In the case of a continuous mapping $f: M \rightarrow N$ between Riemannian manifolds, we may define the condition $f \in W_{\text {loc }}^{1, s}(M, N)$ by the use of local coordinates charts; however, such a procedure is in general not possible for a discontinuous map and we have to proceed differently to define the class of Sobolev mappings between Riemannian manifolds.

We follow the approach of [33], [42].

Definitions. - 1) The mapping $f: M \rightarrow N$ belongs to $L_{\text {loc }}^{s}(M, N)$, $1 \leqslant s \leqslant \infty$, if and only if the function $[f]_{y}: M \rightarrow \mathbb{R}$, defined by $[f]_{y}(x)=d(f(x), y)$, is in $L_{\text {loc }}^{s}(M, \mathbb{R})$ for all point $y \in N$.

2) The map $f$ belongs to $W_{\text {loc }}^{1, s}(M, N)$ if and only if $[f]_{y} \in W_{\text {loc }}^{1, s}(M, \mathbb{R})$ and there exists a function $g \in L_{\text {loc }}^{s}(M, \mathbb{R})$ such that $\left|\nabla[f]_{y}\right|(x) \leqslant g(x)$ a.e. in $M$ for any point $y \in N$.

3) The map $f$ belongs to $A C L_{\mathrm{loc}}^{s}(M, N)$ if it satisfies the following three conditions:

i) the function $M \ni x \rightarrow[f]_{z}(x)=d(f(x), z)$ belongs to $L_{\text {loc }}^{s}(M)$ for every point $z \in N$;

ii) the mapping $f: M \rightarrow N$ is absolutely continuous on lines in the following sense: for any coordinate chart $\varphi: U \rightarrow \mathbb{R}^{n}$ on $M$, the function

$$
(x, \tau) \rightarrow g_{i}(x, \tau):=\operatorname{length}\left(f \circ \varphi^{-1}\left(\left[x, x+\tau \mathbf{e}_{i}\right]\right)\right)
$$

is absolutely continuous in the parameter $\tau$ for all $i$ and almost all $x \in \mathbf{e}_{i}^{\perp}$.

iii) the derivative $\partial_{i} g_{i}: x \rightarrow \lim _{\tau \rightarrow+0} \frac{g_{i}(x, \tau)}{\tau}$, which exists almost everywhere in $U$, belongs to $L_{\text {loc }}^{s}(U)$ for all $i$. 
Proposition 3.1. — The following assertions are equivalent:

1) $f \in W_{\mathrm{loc}}^{1, s}(M, N)$;

2) $f \in A C L_{\mathrm{loc}}^{s}(M, N)$;

3) $f \in L_{\text {loc }}^{s}(M, N)$ and there exists a function $g \in L_{\text {loc }}^{s}(M, \mathbb{R})$ such that for any Lipschitz function $\psi: N \rightarrow \mathbb{R}$, the function $\varphi:=\psi \circ f: M \rightarrow \mathbb{R}$ belongs to $W_{\text {loc }}^{1, s}(M, \mathbb{R})$ and $|\nabla \varphi(x)| \leqslant \operatorname{Lip}(\psi) g(x)$ a.e. in $M$.

4) for any isometric embedding $i: N \rightarrow \mathbb{R}^{k}$ all coordinate functions of the composition $i \circ f$ belong to $W_{\mathrm{loc}}^{1, s}(M, \mathbb{R})$.

Proof. - The proof follows the order $(1) \Rightarrow(2) \Rightarrow(3) \Rightarrow(4) \Rightarrow(2)$. Observe that $(3) \Rightarrow(1)$ is trivial since distance functions are 1-Lipschitz.

Then $(1) \Rightarrow(2)$ and $(2) \Rightarrow(3)$ are proven in [42, Proposition 3] (notice that $(1) \Rightarrow(3)$ is also proven in [33, Theorem 5.1] by other arguments).

The proof of $(4) \Rightarrow(2)$ is given in [34, Proposition 1.2] for the special case $N=\mathbb{R}^{n}$. Its extension to the case of a submanifold $N \subset \mathbb{R}^{k}$ is based on the formula

$$
g_{i}(x, \tau)=\int_{0}^{\tau}\left|\frac{d}{d t}\left(f \circ \varphi^{-1}\left(\left[x, x+t \mathbf{e}_{i}\right]\right)\right)\right| d t
$$

which holds for all absolutely continuous curves in the $\mathbb{R}^{k}$. The general case now follows from the fact that any Riemannian manifold admits an isometric embedding in some Euclidean space.

$(3) \Rightarrow(4)$. We consider an isometric embedding $i: N \rightarrow \mathbb{R}^{k}$ and some coordinate function $z_{j}$ in $\mathbb{R}^{k}$. The restriction $\left.z_{j}\right|_{N}$ is a Lipschitz function on $N$, thus the composition $z_{j} \circ f$ belongs to $W_{\text {loc }}^{1, s}(M, \mathbb{R})$.

The next proposition says that a Sobolev mapping is Lipschitz on a big set.

Proposition 3.2. - Let $f \in W_{\text {loc }}^{1,1}(M, N)$. Then there exists a measurable decomposition $M=E_{f} \cup \cup_{i=1}^{\infty} A_{i}$ such that $\mu\left(E_{f}\right)=0, A_{i}$ is compact for all $i$ and $\left.f\right|_{A_{i}}$ is Lipschitz.

Proof. - Using the previous proposition (assertion 4) we can reduce the proof to the well-known Whitney's approximation theorem for Sobolev function (see e.g. [4, p. 254]). 
As a consequence of this proposition, we have the following version of the change of variables formula for integrals (also known as the area formula), recall that $\chi_{A}$ denotes the characteristic function of a set $A \subset M$.

Proposition 3.3. - Let $f \in W_{\text {loc }}^{1,1}(M, N)$ be a Sobolev mapping between Riemannian manifolds of the same dimension. Then there exists a subset $E_{f} \subset M$ of measure zero such that for all measurable function $\psi: M \rightarrow \mathbb{R}_{+}$we have

$$
\int_{M} \psi(x)\left|J_{f}(x)\right| d \mu(x)=\int_{N}\left(\sum_{f(x)=y} \psi(x) \chi_{M \backslash E_{f}}(x)\right) d \nu(y) .
$$

If $f$ satisfies Lusin's property, then one may take $E=\emptyset$.

See e.g. [11] for a proof.

For the area formula to be useful, we need to work with mappings having a locally integrable Jacobian. Observe in particular that if $f \in$ $W_{\text {loc }}^{1,1}(M, N)$ has bounded $s$-distortion and $J_{f} \in L_{\text {loc }}^{1}(M)$, then we have in fact $f \in W_{\text {loc }}^{1, s}(M, N)$.

The next two lemmas give us sufficient conditions for the local integrability of the Jacobian.

Lemma 3.1. - Let $f: M \rightarrow N$ be a mapping such that $f \in$ $W_{\mathrm{loc}}^{1,1}(M, N)$ and $\left|\Lambda_{n-1} f\right| \in L_{\mathrm{loc}}^{n /(n-1)}(M)$. Then $J_{f} \in L_{\mathrm{loc}}^{1}(M)$.

Proof. - This is a trivial consequence of the inequality $J_{f} \leqslant$ $\left|\Lambda_{n-1} f\right|^{n /(n-1)}$.

LEMMA 3.2. - If $f \in W_{\mathrm{loc}}^{1,1}(M, N)$ is continuous and has essentially finite multiplicity or is open and discrete, then $J_{f} \in L_{\mathrm{loc}}^{1}(M)$.

Proof. — This follows directly from the area formula.

We now give sufficient conditions for Lusin's property:

LEMMA 3.3. - Let $f: M \rightarrow N$ be a mapping satisfying one of the following conditions: $L_{\mathrm{loc}}^{n /(n-1)}(M)$;

1) $f \in W_{\mathrm{loc}}^{1, s}(M, N)$ with $s \geqslant(n-1), J_{f}>0$ a.e. and $\left|\Lambda_{n-1} f\right| \in$

2) $f \in W_{\text {loc }}^{1,1}(M, N)$ is almost absolutely continuous; 
3) $f \in W_{\text {loc }}^{1, n}(M, N)$ is continuous open and discrete.

Then it also satisfies Lusin's property.

Under hypothesis (1) this is Theorem 5.3 in [26]; see also [43] for the case $s=n$. In case (2), this is Theorem 8 from [41]. In case (3), this is a result from [20]; see also [40] for a short proof.

We refer to [23] and [19] for further results on Lusin's condition.

Proposition 3.4. - If the map $f$ is continuous, open and discrete and has bounded $s$-distortion for $s>(n-1)$, then it is differentiable almost everywhere.

See Lemma 4.4 in chapter VI of Rickman's book [34] or Proposition 1 in [41] for a more general result.

Finally we will also need the following result about the exterior differential of the pull-back of a $(n-1)$-form:

Lemma 3.4. - Let $f: M \rightarrow N$ be a mapping satisfying one of the following conditions:

1) $f \in W_{\mathrm{loc}}^{1, n-1}(M, N)$ and $\left|\Lambda_{n-1} f\right| \in L_{\mathrm{loc}}^{n /(n-1)}(M)$;

2) $f$ is almost absolutely continuous, $f \in W_{\text {loc }}^{1, s}(M, N)$ for some $s>(n-1)$ and $J_{f} \in L_{\mathrm{loc}}^{1}(M)$.

Let $\beta$ be a smooth $(n-1)$-form. Then $\alpha:=f^{*} \beta \in L_{\text {loc }}^{1}\left(M, \Lambda^{n-1}\right)$ and

$$
d \alpha=f^{*}(d \beta) .
$$

This result is proved in [26, Th. 3.2] under the first hypothesis and in $[41$, Th. 8$]$ in the case of the second hypothesis

\section{On homeomorphisms with bounded $s$-distortion.}

In this section, we discuss the special case of homeomorphisms with bounded $s$-distortion.

Definition. - The $s$-Dirichlet space of a Riemannian manifold $M$ is the space $\mathcal{L}^{1, s}(M)$ of functions $u \in W_{\text {loc }}^{1, s}(M, \mathbb{R})$ such that $\int_{M}|\nabla u|^{s} d \mu<$ $\infty$. This space is equipped with the semi-norm

$$
\|u\|_{\mathcal{L}^{1, s}(M)}=\|\nabla u\|_{L^{s}(M)} .
$$


If $f: M \rightarrow N$ is a homeomorphism and $v: N \rightarrow \mathbb{R}$ is any function, we denote by $f^{*} v=v \circ f$ its pull back on $M$. If $u: M \rightarrow \mathbb{R}$, we denote by $f_{\sharp} u=u \circ f^{-1}: N \rightarrow \mathbb{R}$ its pushforward.

S. Vodop'yanov has proved the following result [38], [39] (see its generalized version in [44, Theorems 1 and 9]):

THEOREM 4.1. - Let $f: M \rightarrow N$ be a homeomorphism between $n$-dimensional Riemannian manifolds. Fix $s \in[1, \infty)$, then the following assertions are equivalent:

1) $f^{*}: \mathcal{L}^{1, s}(N) \rightarrow \mathcal{L}^{1, s}(M)$ is a bounded operator;

2) $f \in W_{\text {loc }}^{1, s}(M, N)$ and $f$ has bounded s-distortion: $|d f(x)|^{s} \leqslant$ $K J_{f}(x)$ a.e. $x \in M$.

Moreover, if $s \in(1, \infty)$, then condition (1) or (2) are equivalent to

3) $f^{-1}$ decreases the s-capacities of condensers up to a constant:

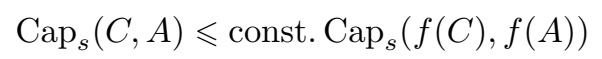

for any condensers $(C, A)$ in $M$.

Finally, if $s>(n-1)$ and Lusin's property holds, then any condition (1)-(3) is equivalent to

4) $f_{\sharp}: \mathcal{L}^{1, p}(M) \rightarrow \mathcal{L}^{1, p}(N)$ is a bounded operator where $p=\frac{s}{s-(n-1)}$, and $\left|d f^{-1}(y)\right|^{p} \leqslant K^{p-1} J_{f^{-1}}(y)$ a.e. $y \in N$, consequently $f^{-1}$ has bounded p-distortion.

Proof. - We only give a short proof of the second part of assertion (4). By Proposition 3.4, the map $f$ is differentiable a.e. and by [44, Theorem 9], we know that $g:=f^{-1}: N \rightarrow M$ is $A C L$ (see also Lemma 5.6 below). Thus we have $d g_{f(x)} \circ d f_{x}=$ Id a.e. in $M$. Notice also that $J_{g}(y) \neq 0$ a.e. in $N$ since $f$ has Lusin's property by hypothesis, we thus have almost everywhere

$$
\left|d g_{f(x)}\right| \leqslant \frac{\left|d f_{x}\right|^{n-1}}{J_{f}(x)}
$$

and therefore

$$
\left|d g_{f(x)}\right|^{p} \leqslant \frac{\left(\left|d f_{x}\right|^{s}\right)^{\frac{p(n-1)}{s}}}{J_{f}^{p}(x)}=\left(\frac{\left|d f_{x}\right|^{s}}{J_{f}(x)}\right)^{p-1} J_{f}^{-1}(x) \leqslant K^{p-1} J_{g}(f(x)) .
$$

A useful consequence of this theorem is the following 
Corollary 4.1. - If $f: M \rightarrow N$ and $g: N \rightarrow W$ are homeomorphisms with bounded s-distortion, then $g \circ f: M \rightarrow W$ also has bounded $s$-distortion.

Special cases of the previous result where also obtained in [9.2 and 12.3], [24], [25, Section 6.4.3] and [30].

Definition. - The Royden algebra of $M$ is the subspace $\mathcal{R}^{s}(M) \subset$ $\mathcal{L}^{1, s}(M)$ of bounded continuous functions; it is a Banach algebra with norm

$$
\|u\|_{\mathcal{R}^{s}}=\|u\|_{L^{\infty}}+\|\nabla u\|_{L^{s}} .
$$

We denote by $K_{\mathcal{R}}$ the norm of the operator $f^{*}: \mathcal{R}^{s}(N) \rightarrow \mathcal{R}^{s}(M)$ and by $K_{\mathcal{L}}$ the norm of the operator $f^{*}: \mathcal{L}^{1, s}(N) \rightarrow \mathcal{L}^{1, s}(M)$.

Proposition 4.1. - Suppose $1<s<\infty$, then for any homeomorphism $f: M \rightarrow N$ we have $K_{\mathcal{R}}=\max \left\{1, K_{\mathcal{L}}\right\}$.

We will need the following

Lemma 4.1. - Let $v \in \mathcal{R}^{s}(N)$ be a non constant function, and fix $\varepsilon>0$. If $1<s<\infty$, then for any $t \in(\alpha, \beta)$, where $\alpha:=\inf v$ and $\beta:=\sup v$, there exists $r=r(t, \varepsilon)>0$ such that $r<\min \{t-\alpha, \beta-t\}$ and

$$
\varepsilon^{-1}\left(t^{\prime \prime}-t^{\prime}\right) \leqslant\left\|\max \left(\min \left(v, t^{\prime \prime}\right), t^{\prime}\right)-t^{\prime}\right\|_{\mathcal{L}^{1, s}(N)}
$$

for all $t^{\prime}, t^{\prime \prime} \in(\alpha, \beta)$ such that $t-r<t^{\prime} \leqslant t \leqslant t^{\prime \prime}<t+r$.

Proof. - Suppose the lemma false, then the function

$$
v_{t^{\prime}, t^{\prime \prime}}:=\frac{\max \left(\min \left(v, t^{\prime \prime}\right), t^{\prime}\right)-t^{\prime}}{t^{\prime \prime}-t^{\prime}}
$$

satisfies $\left\|v_{t^{\prime}, t^{\prime \prime}}\right\|_{\mathcal{L}^{1, s}(N)} \leqslant \frac{1}{\varepsilon}$ for some $\varepsilon>0$ and all $t^{\prime}, t^{\prime \prime} \in(\alpha, \beta)$ such that $t-r<t^{\prime} \leqslant t \leqslant t^{\prime \prime}<t+r$. Consider a bounded domain $A \subset N$ such that $A_{0}:=\{x \in A: v(x)<t\}$ and $A_{1}:=\{x \in A: v(x)>t\}$ are non empty open subsets.

The family $\left\{v_{t^{\prime}, t^{\prime \prime}}\right\}$ is bounded in $W^{1, s}(A)$ and hence weakly compact. It follows that there is a sequence $v_{n}:=v_{t_{n}^{\prime}, t_{n}^{\prime \prime}}$ such that $t_{n}^{\prime} \leqslant t \leqslant t_{n}^{\prime \prime}$ and $\left(t_{n}^{\prime \prime}-t_{n}^{\prime}\right) \rightarrow 0$, which converges weakly to some function $w \in W^{1, s}(A)$. We can furthermore assume that the sequence $\lambda_{n}:=\frac{t-t_{n}^{\prime}}{t_{n}^{\prime \prime}-t_{n}^{\prime}} \in[0,1]$ converges to some number $\lambda$. Using Mazur's Lemma, we can produce convex combinations of the $v_{n}$ converging strongly to $w$. Hence $w=0$ a.e. on $A_{0}$, 
$w=1$ a.e. on $A_{1}$ and $w=\lambda$ a.e. on the level set $A_{t}:=\{x \in A: v(x)=t\}$. But then $\nabla w=0$ a.e. in $A$, hence $w$ is constant a.e. in $A$ which is impossible since $A_{0}$ and $A_{1}$ have positive measure.

Proof of Proposition 4.1. - Observe that $K_{\mathcal{R}} \geqslant 1$ since constant functions belong to the Royden algebras. So we only need to prove the inequalities $K_{\mathcal{L}} \leqslant K_{\mathcal{R}} \leqslant \max \left\{1, K_{\mathcal{L}}\right\}$. Since $f$ is a homeomorphism, $f^{*}$ defines an isometry $f^{*}: L^{\infty}(N) \rightarrow L^{\infty}(M)$ and the inequality $K_{\mathcal{R}} \leqslant$ $\max \left\{1, K_{\mathcal{L}}\right\}$ follows immediately.

To prove the inequality $K_{\mathcal{L}} \leqslant K_{\mathcal{R}}$ it suffices, by density of $\mathcal{R}^{s}(N)$ in $\mathcal{L}^{1, s}(N)$, to show that

$$
\left\|f^{*} v\right\|_{\mathcal{L}^{1, s}(M)} \leqslant(1+\varepsilon) K_{\mathcal{R}}\|v\|_{\mathcal{L}^{1, s}(N)}
$$

for any $\varepsilon>0$ and any function $v \in \mathcal{R}^{s}(N)$.

Set $\alpha:=\inf v$ and $\beta:=\sup v$. By compactness of the interval $[\alpha, \beta]$, we can find a subdivision $\tau=\left\{\alpha=t_{0}<t_{1}<\ldots<t_{l}<t_{l+1}=\beta\right\}$, such that $\left(t_{i+1}-t_{i}\right)<r_{i}$ for $i=1, \ldots, l-1$, where $r_{i}=r(t, \varepsilon)$ satisfies the property of the previous lemma for some $t \in\left(t_{i}, t_{i+1}\right)$.

Set $v_{\tau}:=\alpha+\sum_{i=1}^{l-1} v_{i}$, where $v_{i}:=\max \left(\min \left(v, t_{i+1}\right), t_{i}\right)-t_{i}$. By the lemma we have $\left\|v_{i}\right\|_{L^{\infty}} \leqslant \varepsilon\left\|v_{i}\right\|_{\mathcal{L}^{1, s}(N)}$ for $i=1, \ldots, l-1$, hence

$$
\begin{aligned}
\left\|f^{*} v_{\tau}\right\|_{\mathcal{L}^{1, s}(M)}^{s} & \leqslant\left\|f^{*} v_{\tau}\right\|_{\mathcal{R}^{s}(M)}^{s} \leqslant \sum_{i=0}^{l-1}\left\|f^{*} v_{i}\right\|_{\mathcal{R}^{s}(M)}^{s} \\
& \leqslant \sum_{i=0}^{l-1} K_{\mathcal{R}}^{s}\left(\left\|v_{i}\right\|_{L^{\infty}(N)}+\left\|v_{i}\right\|_{\mathcal{L}^{1, s}(N)}\right)^{s} \\
& \leqslant K_{\mathcal{R}}^{s}(1+\varepsilon)^{s} \sum_{i=1}^{l-1}\left\|v_{i}\right\|_{\mathcal{L}^{1, s}(N)}^{s} \\
& \leqslant K_{\mathcal{R}}^{s}(1+\varepsilon)^{s}\left\|v_{\tau}\right\|_{\mathcal{L}^{1, s}(N)}^{s}
\end{aligned}
$$

because $\left\|v_{\tau}\right\|_{\mathcal{L}^{1, s}(N)}^{s}=\sum_{i=1}^{l-1}\left\|v_{i}\right\|_{\mathcal{L}^{1, s}(N)}^{s}$. The inequality (4) now follows since $\left\|v-v_{\tau}\right\|_{\mathcal{L}^{1, s}(N)} \rightarrow 0$ and $\left\|f^{*} v-f^{*} v_{\tau}\right\|_{\mathcal{L}^{1, s}(N)} \rightarrow 0$ as $\max \left\{t_{1}-\right.$ $\left.t_{0}, t_{l+1}-t_{l}\right\} \rightarrow 0$.

Remark. - Pierre Pansu has defined in [28, p. 475] the notion of homeomorphism of bounded s-dilatation as homeomorphism such that $K_{\mathcal{R}} \leqslant \infty$. It follows from the results of this section that the definition of homeomorphism of bounded $s$-dilatation used by Pansu, coincides with our notion of homeomorphism with bounded $s$-distortion if $1<s<\infty$.

TOME 52 (2002), FASCICULE 6 
It also follows from Theorem 4.1 that if $f$ is a homeomorphism satisfying Lusin's property with bounded $s$-dilatation in Pansu's sense, then $f^{-1}$ is a homeomorphism with bounded $p$-dilatation where $1 / s+(n-1) / p=1$. This gives a positive answer to question 10.3 in [28] in the case where Lusin's property holds.

\section{Pushing functions forward.}

The proof of Theorem A is based on a capacity estimate for the pushforward operator (Corollary 5.1) which is important in itself. It is the goal of this section to prove this capacity estimate.

Let $f: M \rightarrow N$ be a continuous mapping and $u: M \rightarrow \mathbb{R}$ a bounded function. We define the pushforward of $u$ to be the function $v=f_{\sharp} u: N \rightarrow \mathbb{R}$ given by

$$
v(y):= \begin{cases}\sup \{u(x): f(x)=y\} & \text { if } y \in f(M) \\ 0 & \text { otherwise. }\end{cases}
$$

Lemma 5.1. - If $f$ is continuous discrete and open, and $u: M \rightarrow \mathbb{R}$ is continuous with compact support, then the function $v=f_{\sharp} u: N \rightarrow \mathbb{R}$ is also continuous and $\operatorname{supp} v \subset f(\operatorname{supp} u)$.

This is Lemma 7.6 in [22].

If the mapping $f$ has bounded $s$-distortion and $u \in C_{0}^{1}(M, \mathbb{R})$ then $v=f_{\sharp} u$ belongs to $W_{\text {loc }}^{1, p}(N, \mathbb{R})$ where $p=\frac{s}{s-(n-1)}$ provided $s>(n-1)$. More precisely:

Theorem 5.1. - Let $f \in W_{\text {loc }}^{1,1}(M, N)$ be a continuous open and discrete mapping with bounded s-distortion, $(n-1)<s<\infty$. Assume also that $f$ satisfies Lusin's property if $n-1<s<n$. Then the operator $f_{\#}$ possesses the following properties:

1) $f_{\sharp}: C_{0}^{1}(M) \rightarrow W^{1, p}(N) \cap C_{0}^{0}(N)$,

2) $\int_{N}\left|d f_{\sharp}(u)\right|^{p} d \nu \leqslant K^{p-1} \int_{M}|d u|^{p} d \mu$, for any $u \in C_{0}^{1}(M)$. where $p=\frac{s}{s-n+1}$ and $K$ is the constant in (1).

Remarks. - 1) If $f$ is a continuous open mapping and $f \in$ $W_{\text {loc }}^{1, n}(M, N)$, then it always satisfies Lusin's property [20] (see also [40] for a short proof). 
2) This theorem is known for $s=n$ (see [22]). It is also known for general values of $s$ when $f$ is a homeomorphism [44]. Our proof will be based on techniques borrowed from these two papers.

If $f$ is continuous and open, then the image $(f(C), f(A))$ of a condenser $(C, A)$ in $M$ is again a condenser in $N$.

Corollary 5.1. - For any condenser $(C, A)$ in $M$ we have

$$
\left.\operatorname{Cap}_{p}(f(C), f(A))\right) \leqslant K^{p-1} \operatorname{Cap}_{p}(C, A) .
$$

Proof. - Choose a non negative function $u \in C_{0}^{1}(M)$ such that $u=1$ on $C, \operatorname{supp}(u) \subset A$ and $\int_{A}|d u|^{p} \leqslant \operatorname{Cap}_{p}(C, A)+\varepsilon$ where $\varepsilon>0$ is arbitrary.

Let us set $v=f_{\sharp} u: N \rightarrow \mathbb{R}$. Then, by Theorem 5.1 we have $v \in W^{1, p}(A) \cap C_{0}^{0}(A)$. Since $v \geqslant 1$ on $C$, we have

$$
\operatorname{Cap}_{p}(f C, f A) \leqslant \int_{f A}|d v|^{p} \leqslant K^{p-1} \int_{A}|d u|^{p} \leqslant K^{p-1}\left(\operatorname{Cap}_{p}(C, A)+\varepsilon\right) .
$$

We begin the proof of Theorem 5.1 by some lemmas on capacities of condensers:

LEMMA 5.2. - The inequality

$$
\operatorname{Cap}_{s}(C, A) \leqslant \frac{|A|}{\operatorname{dist}(C, \partial A)^{s}}
$$

holds for the capacity of any bounded condenser $(C, A) \subset \mathbb{R}^{n}$.

Proof. — Take $u(x):=\min \left\{\frac{\operatorname{dist}(\partial A, x)}{\operatorname{dist}(\partial A, C)}, 1\right\}$ as a test function.

Lemma 5.3. - Let $(C, A) \subset \mathbb{R}^{n}$ be a condenser such that $C$ is connected. If $(n-1)<s<\infty$, then

$$
\operatorname{Cap}_{s}^{n-1}(C, A) \geqslant b(n, s)(\operatorname{diam} C)^{s}|A|^{(n-1-s)}
$$

where the constant $b(n, s)$ depends on $n$ and $s$ only.

$$
\text { Proof. — See Lemma } 5 \text { of [44]. }
$$


Recall that a domain $\Omega \subset M$ is said to be a normal domain for $f$ if $\bar{\Omega}$ is compact and $\partial(f(\Omega))=f(\partial \Omega)$. For any normal domain $\Omega \subset M$ we have $N_{f}(\Omega)<\infty$. A condenser $(C, A)$ is a normal condenser if $A$ is a normal domain of $f$.

Lemma 5.4. - If $\Omega \subset M$ is a normal domain then $\operatorname{Cap}_{s}(C, A) \leqslant$ $K N_{f}(\Omega) \operatorname{Cap}_{s} f(C, A)$ for any condenser $(C, A)$ in $\Omega$.

This is a direct consequence of Lemma 6.2 below. See also [44, Th. 4].

The next lemma sums up the basic topological properties of a discrete and open mapping $f: M \rightarrow N$. If $x \in M$ and $r>0$, then we denote by $U(x, f, r)$ the connected component of $f^{-1}(B(f(x), r))$ containing $x$.

Lemma 5.5. - Let $f: M \rightarrow N$ be a continuous discrete and open mapping. Then $\lim _{r \rightarrow 0} \operatorname{diam} U(x, f, r)=0$ for every $x \in M$. If $\bar{U}(x, f, r)$ is compact then $U(x, f, r)$ is a normal domain and $f(U(x, f, r))=B(f(x), r)$. Furthermore, for every point $x \in N$ there is a positive number $\sigma_{x}$ such that the following conditions are satisfied for $0<r \leqslant \sigma_{x}$ :

i) $U(x, f, r)$ is a normal neighborhood of $x$,

ii) $U(x, f, r)=U\left(x, f, \sigma_{x}\right) \cap f^{-1}(B(f(x), r))$,

iii) $\partial U(x, f, r)=U\left(x, f, \sigma_{x}\right) \cap f^{-1}(S(f(x), r))$ if $r<\sigma_{x}$,

iv) $M \backslash U(x, f, r)$ is connected if $M$ is connected,

v) $M \backslash \bar{U}(x, f, r)$ is connected if $M$ is connected,

vi) if $0<r<s \leqslant \sigma_{x}$, then $\bar{U}(x, f, r) \subset U(x, f, s)$, and $U(x, f, s) \backslash$ $\bar{U}(x, f, r)$ is a ring.

See [22], [34] or [12] for a proof.

Lemma 5.6. - Let $f: M \rightarrow N$ be as in Theorem 5.1 and $u \in C_{0}^{1}(M)$. Then the function $v=f_{\sharp} u$ is $A C L$.

Recall that a function $v: N \rightarrow \mathbb{R}$ is absolutely continuous on lines $(A C L)$ if for any local parametrization $\varphi: Q \rightarrow N$ (where $Q=\left\{y \in \mathbb{R}^{n}\right.$ : $\left.a_{i} \leqslant y_{i} \leqslant b_{i}\right\} \subset \mathbb{R}^{n}$ is some $n$-interval) and for almost all $z \in P_{k}(Q)$ (= the projection of $Q$ on the hyperplane $\left.y_{k}=0\right)$, the one-variable function $t \rightarrow v\left(\varphi\left(z+t \mathbf{e}_{k}\right)\right)$ is absolutely continuous. 
Proof. - Let us fix some notations. Fix a local parametrization $\varphi: Q \rightarrow N$ (where $Q=\left\{t \in \mathbb{R}^{n}: a_{i} \leqslant t_{i} \leqslant b_{i}\right\} \subset \mathbb{R}^{n}$ is some closed $n$-interval). Choose $Q$ small enough so that for any ball $B(y, r) \subset \varphi(Q)$ the domains $U_{i}:=U\left(x_{i}, f, r\right)$ are disjoint normal neighborhoods of $x_{i}$ for $1 \leqslant i \leqslant q$ where $\left\{x_{1}, \ldots, x_{q}\right\}=f^{-1}(y) \cap \operatorname{supp} u$.

The function $v \circ \varphi^{-1}$ will be simply denoted by $v: Q \rightarrow \mathbb{R}$. We need to show that for any $l=1, \ldots, n$ and for almost all $z \in P_{l}(Q)$, the function $v$ is absolutely continuous on the line segment $\beta_{z}:\left[a_{l}, b_{l}\right] \rightarrow Q$ defined by $\beta_{z}(t)=z+t \mathbf{e}_{l}$.

To this aim, we define a set function $\varphi$ on $P_{l}(Q)$ by

$$
\Phi(A):=\left|U \cap f^{-1}\left(\varphi\left(A \times\left[a_{l}, b_{l}\right]\right)\right)\right|
$$

where $U=\cup_{i=1}^{q} U_{i}$ and $A \subset P_{l}(Q)$ is any Borel set. Then $\Phi$ is a completely additive set function in $P_{l}(Q)$ and from Lebesgue's differentiation theorem, we know that $\Phi^{\prime}(z)<\infty$ for almost all $z \in P_{l}(Q)$.

It is known (see [22, Lemma 2.7]) that for every point $x_{0} \in U \cap$ $f^{-1}\left(z+a_{l} \mathbf{e}_{l}\right)$ there exists a path $\alpha:\left[a_{l}, b_{l}\right] \rightarrow U$ such that $\alpha\left(a_{l}\right)=x_{0}$ and $f \circ \alpha=\varphi \circ \beta_{z}$. We call such a path a lift of $\beta_{z}(t)=z+t \mathbf{e}_{l}$ with base point $x_{0}$; clearly the number of lifts does not exceed $N_{f}(U)$.

Claim. - Let $\alpha:\left[a_{l}, b_{l}\right] \rightarrow U$ be any lift of $\beta_{z}$. If $\Phi^{\prime}(z)<\infty$, then $\alpha$ is absolutely continuous.

Since the $A C L$-property is local it suffices to show that $\alpha$ is $A C L$ in a neighborhood of every point. We may thus restrict our considerations to the case of mappings $f: U \rightarrow Q$ where $U$ is a bounded domain in $\mathbb{R}^{n}$.

To prove the claim, we fix some arbitrary pairwise disjoint closed segments $\Delta_{1}, \ldots, \Delta_{k} \subset\left(a_{l}, b_{l}\right)$ of lengths $b_{1}, \ldots, b_{k}$. Choose $r>0$ small enough so that the sets

$$
R_{i}:=\left\{y \in \mathbb{R}^{n} \mid \operatorname{dist}\left(y, \Delta_{i}\right)<r\right\}
$$

are pairwise disjoint. Let $T_{i}:=\bigcup_{z \in \Delta_{i}} U(\alpha(z), f, r)$, then $\left(\alpha\left(\Delta_{i}\right), T_{i}\right)$ and $\left(\Delta_{i}, R_{i}\right)$ are condensers and $\left(\Delta_{i}, R_{i}\right)=\left(f\left(\alpha\left(\Delta_{i}\right)\right), f\left(T_{i}\right)\right)$; indeed, we have $f\left(\alpha\left(\Delta_{i}\right)\right)=\Delta_{i}$ and

$$
f\left(T_{i}\right)=f\left(\bigcup_{z \in \Delta_{i}} U(\alpha(z), f, r)\right)=\bigcup_{z \in \Delta_{i}} B(z, f, r)=R_{i} .
$$

From Lemmas 5.2 and 5.3, we have

$$
\operatorname{Cap}_{s}\left(\Delta_{i}, R_{i}\right) \leqslant \frac{\left|R_{i}\right|}{r^{s}} \leqslant c_{1} b_{i} r^{n-1-s}
$$

TOME 52 (2002), FASCICULE 6 
and

$$
\operatorname{Cap}_{s}\left(\alpha\left(\Delta_{i}\right), T_{i}\right) \geqslant c_{2} \frac{\left(\operatorname{diam} \alpha\left(\Delta_{i}\right)\right)^{s /(n-1)}}{\left|T_{i}\right|^{(1-n+s) /(n-1)}} .
$$

These inequalities, together with Lemma 5.4, imply

$$
\operatorname{diam} \alpha\left(\Delta_{i}\right) \leqslant c_{3} b_{i}^{\frac{n-1}{s}}\left(\frac{\left|U \cap T_{i}\right|}{r^{n-1}}\right)^{\frac{1-n+s}{s}}
$$

where the constant $c_{3}$ depends on previous constants, $K$ and $N_{f}(\operatorname{supp} u)$.

Set $E(z, r)=\left\{y \in Q: \operatorname{dist}\left(y, \beta_{z}\left(\left[a_{i}, b_{i}\right]\right)\right)<r\right\}$, then $\bigcup_{i=1}^{k} T_{i} \subset$ $f^{-1}(E(z, r))$. Summing the previous inequality over $i=1, \ldots, k$ and applying Hölder's inequality we obtain

$$
\sum_{i=1}^{k} \operatorname{diam} \alpha\left(\Delta_{i}\right) \leqslant c_{4}\left(\frac{\left|U \cap f^{-1}(E(z, r))\right|}{r^{n-1}}\right)^{\frac{1-n+s}{s}}\left(\sum_{i=1}^{k} b_{i}\right)^{\frac{n-1}{s}} .
$$

Letting $r \rightarrow 0$, we find that

$$
\sum_{i=1}^{k} \operatorname{diam} \alpha\left(\Delta_{i}\right) \leqslant c_{5} \varphi^{\prime}(z)\left(\sum_{i=1}^{k} b_{i}\right)^{\frac{n-1}{s}},
$$

hence $\alpha$ is absolutely continuous if $\varphi^{\prime}(z)<\infty$.

We now conclude the proof of the lemma as follows: Let $\alpha_{1}, \alpha_{2}, \ldots \alpha_{d}$ be all the lifts of the segment $\beta_{z}$. If $\Phi^{\prime}(z)<\infty$, then $u \circ \alpha_{i}$ is absolutely continuous since $u$ is $C^{1}$ and $\alpha_{i}$ is absolutely continuous. We conclude that $v \circ \beta_{z}$ is absolutely continuous since

$$
v \circ \beta_{z}=\max _{i} u \circ \alpha_{i} .
$$

Lemma 5.7. - Let $f: M \rightarrow N$ be as in Theorem 5.1, then $J_{f}=0$ almost everywhere on the branch set and the image of the branch set has measure zero.

Proof. - Because $f$ has bounded $s$-distortion and $s>(n-1)$, $f \in W_{\text {loc }}^{1, s}$, it then follows from 3.4 that $f$ is differentiable almost everywhere.

Suppose that $f$ is differentiable at $x$ and $J_{f}(x)>0$, then the index $j(x, f)=1$ (because the map is continuous open and discrete and the topological degree is stable under homotopy, see e.g. pp. 15-21 in [34]).

If $j(x, f)=1$, then $x \notin B_{f}$ (see [34, Proposition 4.10]); it follows that $J_{f}=0$ a.e. on $B_{f}$. 
Because $f$ is assumed to satisfy Lusin's property, we can use the area formula (Proposition 3.3) to conclude that $f\left(B_{f}\right)$ has measure zero:

$$
\nu\left(f\left(B_{f}\right)\right) \leqslant \int_{N}\left(\sum_{f(x)=y} \chi_{B_{f}(x)}\right) d \nu(y)=\int_{M} \chi_{B_{f}(x)} J_{f}(x) d \mu(x)=0 .
$$

Proof of Theorem 5.1. - To conclude the proof of the theorem it only remains to check the integrability of $d v$. To do this we first observe that Vitali's covering Theorem implies

$$
\operatorname{supp} v \backslash f\left(B_{f} \cap \operatorname{supp} u\right) \subset \bigcup_{i=1}^{\infty} B\left(y_{i}, r_{i}\right) \cup A,
$$

where $B_{f}$ is the branch set of $f, A \subset N$ is a set with $\nu(A)=0$ and $B\left(y_{i}, r_{i}\right)$, $i \in \mathbb{N}$, are mutually disjoint balls small enough so that the components of $f^{-1}\left(B\left(y_{i}, r_{i}\right)\right)$ which meet the support of $u$ form a finite disjoint collection $D_{i_{1}}, D_{i_{2}}, \ldots, D_{i_{k}}$ of open subsets of $M$ such the restrictions of $f$ define homeomorphisms $f_{j}: D_{i_{j}} \rightarrow B\left(y_{i}, r_{i}\right), j=1, \ldots k$.

By Theorem 4.1, the inverse of $f_{i}$, i.e. the map $g_{j}:=f_{j}^{-1}: B\left(y_{i}, r_{i}\right) \rightarrow$ $D_{i_{j}}$ is $A C L$, furthermore, we have $\left|d g_{j}\right|^{p} \leqslant K^{p-1} J_{g_{j}}$ a.e. Hence we obtain

$$
|d v(z)|^{p} \leqslant \max _{1 \leqslant j \leqslant k}\left|d u\left(g_{j}(z)\right)\right|^{p}\left|d g_{j}(z)\right|^{p} \leqslant K^{p-1} \sum_{j=1}^{k}\left|d u\left(g_{j}(z)\right)\right|^{p} J_{g_{i}}(z)
$$

for almost every $z \in B\left(y_{i}, r_{i}\right)$. This implies

$$
\begin{gathered}
\int_{B\left(y_{i}, r_{i}\right)}|d v(z)|^{p} d \nu \leqslant K^{p-1} \sum_{j=1}^{k} \int_{B\left(y_{i}, r_{i}\right)}\left|d u\left(g_{j}(z)\right)\right|^{p} J\left(z, g_{j}\right) d \nu \leqslant K^{p-1} . \\
\int_{f^{-1}\left(B\left(y_{i}, r_{i}\right)\right)}|d u|^{p} d \mu .
\end{gathered}
$$

From Lemma 5.7, we know that $\nu\left(f\left(B_{f}\right)\right)=0$ and $J_{f}=0$ a.e. on $B_{f}$; we thus have from the area formula

$$
\begin{aligned}
\int_{N}|d v(z)|^{p} d \nu & =\sum_{i=1}^{\infty} \int_{B\left(y_{i}, r_{i}\right)}|d v(z)|^{p} d \nu \leqslant K^{p-1} \sum_{i=1}^{\infty} \int_{f^{-1}\left(B\left(y_{i}, r_{i}\right)\right)}|d u|^{p} d \mu \\
& \leqslant K^{p-1} \int_{M}|d u|^{p} d \mu .
\end{aligned}
$$

TOME 52 (2002), FASCICULE 6 


\section{Proofs of the main theorems.}

\subsection{Proof of Theorem A.}

Let us recall the statement :

Theorem A. - Let $f \in W_{\text {loc }}^{1,1}(M, N)$ be a continuous open and discrete mapping with bounded $s$-distortion where $s>(n-1)$. Assume also that $f$ satisfies Lusin's property. If $M$ is $p$-parabolic with $p=\frac{s}{s-(n-1)}$, then $N$ is also p-parabolic.

Proof. - Let $D \subset M$ be a compact subset with non empty interior. Because $f$ is a continuous and open map, $f(D) \subset N$ is also a compact set with non empty interior. By Corollary 5.1 we have

$$
\operatorname{Cap}_{p}(f(D), N) \leqslant \operatorname{Cap}_{p}(f(D), f M) \leqslant K^{p-1} \operatorname{Cap}_{p}(D, M),
$$

hence if $M$ is $p$-parabolic then so is $N$.

\subsection{Proofs of Theorems $\mathrm{C}$ and B.}

The proofs will use the following criterion for hyperbolicity which is due to V. Gol'dshtein and M. Troyanov (see [9]).

Theorem 6.1. - Let $M$ be an oriented connected Riemannian manifold $M$. Then the following are equivalent $\left(\frac{1}{p}+\frac{1}{q}=1\right)$ :

1) $M$ is p-hyperbolic;

2) there exists a smooth form $\alpha \in L^{q}\left(M, \Lambda^{n-1}\right)$ such that $d \alpha \geqslant 0$ and $\int_{M} d \alpha \neq 0$

3) there exists a form $\alpha \in L^{q}\left(M, \Lambda^{n-1}\right)$ such that $d \alpha \geqslant 0$ and $\int_{M} d \alpha \neq 0$

4) $H_{\text {comp }, q}^{n}(M)=0$.

The cohomology space $H_{\text {comp }, q}^{n}(M)$ is the space of all closed differential forms of degree $n$ with compact support modulo the differential of $(n-1)$-forms in $L^{q}$. 
We will also need the following lemma :

LEMma 6.1. - Let $f: M \rightarrow N$ be a mapping of class $W_{\text {loc }}^{1,1}$ with essentially finite multiplicity and bounded q-codistortion: $\left|\Lambda_{n-1} f\right|^{q} \leqslant K J_{f}$. Then

$$
\Lambda_{n-1} f: L^{q}\left(N, \Lambda^{n-1}\right) \rightarrow L^{q}\left(M, \Lambda^{n-1}\right)
$$

is a bounded operator with norm $\leqslant\left(K \cdot N_{f}(M)\right)^{1 / q}$.

(Recall that $N_{f}(A)=\operatorname{ess~sup}_{y} \operatorname{Card}\left(A \cap f^{-1}(y)\right)$ for any set $A \subset M$.)

Proof. - Let $\beta \in L^{q}\left(N, \Lambda^{n-1}\right)$, then

$$
\begin{aligned}
\int_{M}\left|\Lambda_{n-1} f(\beta)\right|^{q} d \mu & \leqslant K \int_{M}\left|\beta_{f(x)}\right|^{q} J_{f}(x) d \mu \\
& =K \int_{N}\left(\sum_{f(x)=y}\left|\beta_{f(x)}\right|^{q} \chi_{M \backslash E_{f}}(x)\right) d \nu \\
& \leqslant\left(K \cdot N_{f}(M)\right) \int_{N}|\beta|^{q} d \nu .
\end{aligned}
$$

We now prove Theorem C; we restate it in the following form:

Theorem C. - Let $f: M \rightarrow N$ be a mapping of essentially finite multiplicity with bounded $q$-codistortion where $q>1$ and such that $J_{f}>0$ on some set of positive measure. Assume furthermore either

1) $f \in W_{\mathrm{loc}}^{1, n-1}(M, N)$ and $\left|\Lambda_{n-1} f\right| \in L_{\mathrm{loc}}^{n /(n-1)}(M)$, or

2) $f$ is almost absolutely continuous, $f \in W_{\mathrm{loc}}^{1, s}(M, N)$ for some $s>(n-1)$ and $J_{f} \in L_{\text {loc }}^{1}(M)$.

If $N$ is $p$-hyperbolic with $p=\frac{q}{q-1}$, then $M$ is also $p$-hyperbolic

Proof. - Let us choose a bounded Borel set $U \subset M$ such that $U$ has positive measure, $f(U)$ is bounded and $J_{f}>0$ on $U$. Observe that, by the area formula, $\nu(f(U))>0$.

Choose a non negative smooth function $h: N \rightarrow \mathbb{R}$ with compact support and such that $h>0$ in a neighborhood of $f(U)$. Since $N$ is $p$-hyperbolic, $H_{\text {comp }, q}^{n}(N)=0$, hence there exists an $(n-1)$-form $\beta \in$ $L^{q}\left(N, \Lambda^{n-1}\right)$ such that $d \beta=h \cdot \omega_{N}\left(\omega_{M}\right.$ and $\omega_{N}$ are the volume forms of $M$ and $N$ respectively).

TOME 52 (2002), FASCICULE 6 
By Lemma 6.1 , we have $\alpha:=f^{*} \beta \in L^{q}\left(M, \Lambda^{n-1}\right)$. We then have from Lemma 3.4

$$
d \alpha=f^{*}(d \beta)=(h \circ f) \cdot f^{*} \omega_{N}=(h \circ f) \cdot J_{f} \omega_{M} .
$$

Thus $d \alpha \geqslant 0$ and $\int_{M} d \alpha \geqslant \int_{U}(h \circ f) \cdot J_{f} d \mu>0$ and we conclude by Theorem 6.1 that $M$ is $p$-hyperbolic.

Finally, we deduce Theorem B from Theorem C.

Theorem B. - Let $f \in W_{\mathrm{loc}}^{1, s}(M, N)$ be a mapping of essentially finite multiplicity with bounded $s$-distortion where $s>(n-1)$. Assume either

1) $\left|\Lambda_{n-1} f\right| \in L_{\mathrm{loc}}^{n /(n-1)}(M)$, or

2) $f$ is almost absolutely continuous and $J_{f} \in L_{\text {loc }}^{1}(M)$.

If $M$ is $p$-parabolic and $N$ is p-hyperbolic with $p=s /(s-(n-1))$, then $f$ is constant a.e.

Proof. - Let $q=p /(p-1)$. Then $s=q(n-1)$ and from Lemma 2.1 we know that if $f$ has bounded $s$-distortion, then it has bounded $q$ codistortion. Hence by Theorem $\mathrm{C}$, we have $J_{f}=0$ a.e. and thus $|d f|=0$ a.e. since $|d f|^{s} \leqslant K J_{f}$. As $f$ is a Sobolev mapping, we conclude that $f$ is constant a.e.

\subsection{Proof of Theorem D.}

Lemma 6.2. - Let $f \in W_{\text {loc }}^{1,1}(M, N)$ be a mapping with bounded $s$-distortion and essential finite multiplicity. Then $f^{*}: \mathcal{L}^{1, s}(N) \rightarrow \mathcal{L}^{1, s}(M)$ is a bounded operator with operator norm at most $\left(K N_{f}(M)\right)^{1 / s}$.

Proof. - Let us first consider a function $v \in C^{1}(N) \cap \mathcal{L}^{1, s}(M)$. Then $u:=f^{*} v \in W_{\mathrm{loc}}^{1,1}(M)$ and $d u_{x}=d f_{x}^{t}\left(d v_{f(x)}\right)$. Hence we have almost everywhere $|d u|^{s} \leqslant|d v|^{s}|d f|^{s} \leqslant K|d v|^{s} J_{f}$. From the area formula we thus obtain

$$
\begin{aligned}
\int_{M}\left|d u_{x}\right|^{s} d \mu(x) & \leqslant K \int_{M}\left|d v_{f(x)}\right|^{s} J_{f}(x) d \mu(x) \\
& =K \int_{N}\left(\sum_{f(x)=y}\left|d v_{f(x)}\right|^{s} \chi_{M \backslash E_{f}}(x)\right) d \nu(y) \\
& \leqslant K N_{f}(M) \int_{N}|d v|^{s} d \nu(y)
\end{aligned}
$$


Thus $u \in \mathcal{L}^{1, s}(M)$ and $\|u\|_{\mathcal{L}^{1, s}(M)} \leqslant\left(K N_{f}(M)\right)^{1 / p}\|v\|_{\mathcal{L}^{1, s}(N)}$.

Using the argument on page 673 of [44], we can extend this estimate from functions $v \in C^{1}(N) \cap \mathcal{L}^{1, s}(M)$ to all functions $v \in \mathcal{L}^{1, s}(N)$. This proves that the norm of the operator $f^{*}: \mathcal{L}^{1, s}(N) \rightarrow \mathcal{L}^{1, s}(M)$ is bounded by $\left(K N_{f}(M)\right)^{1 / s}$.

Recall the statement of Theorem D:

Theorem D. - Let $f \in W_{\text {loc }}^{1,1}(M, N)$ be a continuous non constant proper mapping with bounded s-distortion of finite essential multiplicity. If $N$ is $s$-parabolic then so is $M$.

Proof. - Let $D \Subset M$ be a compact set; then $D^{\prime}=f(D) \subset N$ is also compact and, by hypothesis, it has zero $p$-capacity. For each $\varepsilon>0$, one can thus find a continuous function $v \in \mathcal{L}^{1, s}(N)$ with compact support and such that $v \equiv 1$ on $D^{\prime}$ and $\int_{N}|d v|^{s} \leqslant \varepsilon$.

Since $f$ is a proper map, the function $u:=f^{*}(v)$ also has compact support and, clearly, $u \equiv 1$ on $D$. Let $A$ be the norm of the operator $f^{*}: \mathcal{L}^{1, s}(N) \rightarrow \mathcal{L}^{1, s}(M)$; we know by Lemma 6.2 that $A$ is finite. We then have $\int_{M}|d u|^{s} \leqslant A^{s} \int_{N}|d v|^{s} \leqslant A^{s} \varepsilon$. Hence $D$ has zero $p$-capacity and we conclude that $M$ is $p$-parabolic.

\section{Complements.}

\subsection{A topological result.}

A famous theorem of Yu. Reshetnyak states that a non constant quasiregular mapping is open and discrete. We formulate below a generalization of this theorem established recently by S. Vodop'yanov's in [41], which provides topological properties for mappings with integrable distortion.

Theorem 7.1. - Let $f \in W_{\text {loc }}^{1,1}(M, N)$ be a continuous non constant mapping with nonnegative Jacobian $J_{f}(x) \geqslant 0$ and $K(x)=\frac{\left|d f_{x}\right|^{n}}{J_{f}(x)} \in$ $L_{\mathrm{loc}}^{p}(M)$ for some $n-1<p \leqslant \infty$. Assume either

1) $\left|\Lambda_{n-1} f\right| \in L_{\text {loc }}^{n /(n-1)}(M)$, or

2) $f$ is almost absolutely continuous and $J_{f} \in L_{\text {loc }}^{1}(M)$.

Then $f$ is discrete and open. 
Remarks. - 1) If the manifolds are two-dimensional, then the condition $n-1<p \leqslant n$, can be relaxed to $1 \leqslant p \leqslant 2$.

(2) This result was also proven in [13] and [21] under the assumption $f \in W_{\text {loc }}^{1, n}(M, N)$. It has been also recently proved in [18] under different analytical assumptions.

As a consequence of Theorem 7.1 we obtain topological properties for mappings with bounded $s$-distortion. The next assertion gives a positive answer to the question 10.8 of [28].

Corollary 7.1. - Let $f \in W_{\text {loc }}^{1,1}(M, N)$ be a continuous non constant mapping with bounded $s$-distortion where $n-1<s \leqslant n$. Assume either

1) $\left|\Lambda_{n-1} f\right| \in L_{\mathrm{loc}}^{n /(n-1)}(M)$, or

2) $f$ is almost absolutely continuous and $J_{f} \in L_{\text {loc }}^{1}(M)$.

Then $f$ is discrete and open.

Remark. - This result does not hold if $s>n$. Consider for instance the map $f: \mathbb{R}^{n} \rightarrow \mathbb{R}^{n}$ given by

$$
f(x)= \begin{cases}0 & \text { if }|x| \leqslant 1, \\ || x|-1|^{\alpha} \frac{x}{|x|} & \text { if } 1 \leqslant|x| \leqslant 2, \\ || x|-1| \frac{x}{|x|} & \text { if }|x| \geqslant 2,\end{cases}
$$

for some $\alpha>1$. Then $f$ is Lipschitz and has bounded $s$-distortion for $s=\frac{n \alpha-1}{\alpha-1}>n$. Clearly $f$ is neither open nor discrete; however $f$ has finite essential multiplicity.

Proof. - We suppose that $|d f|^{s} \leqslant C J_{f}$ a.e. for some $n-1<s<n$. Let us define the function

$$
K_{f}(x)= \begin{cases}\frac{\left|d f_{x}\right|^{n}}{J_{f}(x)} & \text { if } \quad J_{f}(x) \neq 0 \\ 1 & \text { else. }\end{cases}
$$

Set $p=\frac{s}{n-s}$, we have at almost all points where $J_{f}(x) \neq 0$,

$$
\left|K_{f}\right|^{p}=\frac{|d f|^{n p}}{J_{f}^{p}} \leqslant C^{n p / s} \frac{J_{f}^{n p / s}}{J_{f}^{p}}=C^{n p / s} J_{f}^{p(n / s-1)} \leqslant C^{n p / s} J_{f} .
$$

Thus $K_{f} \in L_{\text {loc }}^{p}$. Since $n-s<1$, we have $p>s>n-1$ and we can conclude the proof from Theorem 7.1. 
Corollary 7.2. - Let $f \in W_{\mathrm{loc}}^{1,1}(M, N)$ be a mapping with bounded $q$-codistortion where

$$
\frac{(n-1)^{2}}{1+(n-1)(n-2)}<q \leqslant \frac{n(n-1)}{1+n(n-2)} .
$$

Assume that $J_{f}>0$ a.e. and either

1) $\left|\Lambda_{n-1} f\right| \in L_{\mathrm{loc}}^{n /(n-1)}(M)$, or

2) $f$ is almost absolutely continuous and $J_{f} \in L_{\text {loc }}^{1}(M)$.

Then $f$ is discrete and open.

Proof. - By Lemma 2.1, $f$ has bounded $s$-distortion for $s=$ $\frac{q}{(n-1)-q(n-2)}$; observe that the inequalities (5) are equivalent to $n-1<$ $s \leqslant n$. Thus the corollary follows from Corollary 7.1.

\subsection{On $p$-parabolic manifolds.}

A connected oriented Riemannian $n$-manifold $M$ is called $p$-parabolic, $1 \leqslant p<\infty$, if $\operatorname{Cap}_{p}(C, M)=0$ for all compact subsets $C \Subset M$ and $p$-hyperbolic otherwise. In this section, we list some facts concerning $p$ parabolicity. We refer to [37], [10], and [45] for further information on this notion.

a) If $M$ contains one compact subset with nonempty interior having zero $p$-capacity then $M$ is $p$-parabolic.

b) The Euclidean space $\mathbb{R}^{n}$ is $p$-hyperbolic for $p<n$ and $p$-parabolic for any $p \geqslant n$.

c) If $M$ is $p$-hyperbolic, then any domain $\Omega \subset M$ is also $p$-hyperbolic.

d) If a closed subset $S \subset M$ with Hausdorff dimension $>(n-p)$ is removed from any manifold $M$ and if $M \backslash S$ is connected, then $M \backslash S$ is $p$-hyperbolic.

e) In particular, if one removes a point $x_{0}$, then $M \backslash\left\{x_{0}\right\}$ is $p$-hyperbolic for all $p>n$ and if one removes a non separating closed subset with nonempty interior $D \subset M$, then $M \backslash D$ is $p$-hyperbolic for all $p \geqslant 1$.

f) If the manifold is complete and $\operatorname{Vol}\left(B\left(x_{0}, r\right)\right) \leqslant$ const. $r^{d}$ then $M$ is $p$-parabolic for any $p \geqslant d$ (finer estimates relating the volume growth to parabolicity are in fact available). 
g) If the isoperimetric inequality

$$
\operatorname{Area}(\partial \Omega)^{d /(d-1)} \geqslant \text { const. } \operatorname{Vol}(\Omega)
$$

holds for any big smooth domain $\Omega \subset M$, then $M$ is $p$-hyperbolic for $p<d$.

h) Suppose that a Sobolev inequality

$$
\|u\|_{L^{q}} \leqslant \text { const. }\|\nabla u\|_{L^{p}}
$$

holds for some $1 \leqslant q \leqslant \infty$ and all functions $u \in C_{0}^{1}(M)$. Then $M$ is p-hyperbolic.

Recall that the $p$-Laplacian is the operator $\Delta_{p} u:=\operatorname{div}\left(|\nabla u|^{p-2} \nabla u\right)$. A function is called $p$-superharmonic if $\Delta_{p} u \leqslant 0$.

i) $M$ is $p$-parabolic if and only if every positive $p$-superharmonic function on $M$ is constant.

j) $M$ is $p$-hyperbolic if and only if there exists a positive Green function for the $p$-Laplacian.

k) $M$ is 2-hyperbolic if and only if the Brownian motion is transient.

l) If $M$ has finite volume, then there exists a number $d \in[1, \infty]$ such that $M$ is $p$-parabolic for $1 \leqslant p<d$ and $p$-hyperbolic for $p>d$.

m) For a non compact manifold with bounded geometry, we have the opposite behaviour: there exists a index $d$, called the parabolic dimension of $M$, such that $M$ is $p$-hyperbolic for $1 \leqslant p<d$ and $p$-parabolic for $p>d$.

n) The parabolic dimension is a quasi-isometric invariant of manifolds with bounded geometry.

o) $n$-parabolicity is a quasi-conformal invariant for any manifolds.

Proof. - The proofs of (a)-(h) and (l)-(n) can be found in [37]. The proofs of (i) and (j) are in [14] (see also [17]). We refer to [10] for $(\mathrm{k})$ and [45] for (o).

\subsection{An improvement of a result by Pierre Pansu.}

The following result gives an improvement of our Theorem B for Sobolev homeomorphisms with Lusin's property between manifolds with 
bounded geometry. It was proved by P. Pansu for diffeomorphisms, see [28, corollaire 2.1].

Theorem. - Let $M$ and $N$ be Riemannian manifolds with bounded geometry, and assume that $N$ satisfies an isoperimetric inequality of order $d>n$ :

$$
\operatorname{Area}(\partial \Omega)^{d /(d-1)} \geqslant \text { const. } \operatorname{Vol}(\Omega)
$$

for all smooth compact domain $\Omega \subset N$ of volume $\geqslant 1$ (in particular $N$ is $n$-hyperbolic).

If $d \frac{n-1}{d-1}<s<n$, then every homeomorphism $f \in W_{\mathrm{loc}}^{1,1}(M, N)$ with bounded $s$-distortion satisfying Lusin's property is a rough quasi-isometry.

Proof. - We know that if $f$ has bounded $s$-distortion, $s>(n-1)$ and satisfies Lusin's property, then $f^{-1}$ has bounded $p$-distortion where $p=\frac{s}{s-(n-1)}$ (see Theorem 4.1 and the Remark at the end of Section 4). The above theorem thus follows from [28, Théorème 1].

\subsection{On Reshetnyak's proof for the case of quasi-regular mappings.}

In order to illustrate the alternative approach based on methods of non-linear potential theory, we give a short proof of Liouville's theorem for quasi-regular mappings along Reshetnyak's ideas.

THEOREM. - Let $f: M \rightarrow N$ be a non constant quasi-regular mapping between oriented $n$-dimensional Riemannian manifolds. Assume that $M$ is $n$-parabolic, then so is $N$.

Proof. - Assume that $f: M \rightarrow N$ is a non constant quasi-regular mapping, then it is known (see [31, Th. 6.4, chap. II]) that $f$ is an open map; in particular $N^{\prime}:=f(M) \subset N$ is open. If $N$ is $n$-hyperbolic, then so is $N^{\prime}$ and, by [14, Th. 5.2], we know that there exists a non constant positive $n$ superharmonic function $v: N^{\prime} \rightarrow \mathbb{R}$. The function $u=f^{*} v=v \circ f: M \rightarrow \mathbb{R}$ is then $\mathcal{A}$-superharmonic where $\mathcal{A}$ is the pull back to $M$ of the operator $T N^{\prime} \rightarrow T N^{\prime}$ given by $\eta \rightarrow|\eta|^{n-2} \eta$ (see [31, Th. 11.2, chap. II] or [12, Th. 14.42]). By [14, Th. 5.2] again, one concludes that $M$ is also $n$-hyperbolic, contradicting the hypothesis.

TOME 52 (2002), FASCICULE 6 
Final remarks. — 1) The argument of Martio, Väisälä and Rickman are based on capacity estimates in the spirit of our proof of Theorem A (see [22]).

2) Another proof can be found in [3]. This paper gives other obstructions to the existence of quasi-regular mappings.

\section{BIBLIOGRAPHY}

[1] M. Chamberland and G. Meister, A Mountain Pass to the Jacobian Conjecture, Canad. Math. Bull., Vol. 41 (1998), 442-451.

[2] V. M. Chernikov and S. K. Vodop'Yanov, Sobolev Spaces and Hypoelliptic Equations, II, Siberian Advances in Mathematics, 6 (1996), 64-96.

[3] T. Coulhon, I. Holopainen and L. Saloff-Coste, Harnack Inequality and hyperbolicity for subelliptic $p$-Laplacians with applications to Picard type theorems, Geom. Funct. Anal., 11 (2001), 1139-1191.

[4] L. C. Evans and R. F. Gariepy, Measure Theory and Fine properties of Functions, Studies in Advanced Mathematics, CRC press (1992).

[5] H. Federer, Geometric Measure Theory, Grundlehren der Mathematik, 153 (1969).

[6] J. Lelong-Ferrand, Étude d'une classe d'applications liées à des homomorphismes d'algèbres de fonctions et généralisant les quasi-conformes, Duke Math. J., 40 (1973), 163-186.

[7] K. Gafaïti, Algèbre de Royden et Homéomorphismes p-dilatation bornée entre espaces métriques mesurés, Thèse, EPFL Lausanne (2001).

[8] V. Gol'dshtein, L. Gurov and A. Romanov, Homeomorphisms that induce Monomorphisms of Sobolev Spaces, Israel J. of Math., 91 (1995), 31-60.

[9] V. Gol'dshtein and M. Troyanov, The Kelvin-Nevanlinna-Royden criterion for p-parabolicity, Math. Zeitschrift, 232 (1999), 607-619.

[10] A. Grigor'YAn, Analytic and Geometric Background of Recurence and NonExplosion of the Brownian Motion on Riemannian Manifolds, Bull. Amer. Math. Soc., 36 (1999), 135-242.

[11] P. HajłAsz, Change of Variables Formula under Minimal Assumptions, Colloquium Mathematicum, 64 (1993), 93-101.

[12] J. Heinonen, T. Kilpeläinen and O. Martio, Non Linear Potential Theory of Degenerate Elliptic Equations, Oxford Math. Monographs (1993).

[13] J. Heinonen and P. Koskela, Sobolev Mappings with Integrable Dilatation, Arch. Rational Mech.Anal., 125 (1993), 81-97.

[14] I. Holopainen, Non linear Potential Theory and Quasiregular Mappings on Riemannian Manifolds, Annales Academiae Scientiarum Fennicae Series A, 74 (1990).

[15] I. Holopainen, Rough isometries and $p$-harmonic functions with finite Dirichlet integral, Revista Mathemática Iberoamericana, 10 (1994), 143-175.

[16] T. Iwaniec, P. Koskela and J. Onninen, Mappings of finite distortion: Monotonicity and continuity, Preprint. 
[17] M. KAnAI, Rough isometries and the parabolicity of Riemannian manifolds, J. Math. Soc. Japan, 38, n⿳ 2 (1986), 227-238.

[18] J. Kauhanen, P. Koskela and J. Malỳ, Mappings of finite distortion: discreteness and openess, University of Jyvت̈askylä (2000), preprint 226.

[19] J. Malỳ, Absolutely continuous functions of several variables, J. Math. Anal. Appl., 231 (1999), 492-508.

[20] J. MALỲ and O. MARTiO, Lusin's condition (N) and mappings of the class $W^{1, n}$, J. Reine Angew. Math., 458 (1995), 19-36.

[21] J. J. Manfredi and E. Villamor, An extension of Reshetnyak's theorem, Indiana Univ. Math. J., 47, n⿳0 3 (1998), 1131-1145.

[22] O. Martio, S. Rickman and J. VÄIsÄLÄ, Definitions for Quasiregular Mappings, Ann. Acad. Sc. Fenn., 448 (1969), 5-40.

[23] O. Martio and W. P. Ziemer, Lusin's condition (N) and mappings of non negative Jacobian, Michigan. Math. J., 39 (1992), 495-508.

[24] V.G. MAZ'YA, Sobolev Spaces, Springer Verlag, 1985.

[25] V. Maz'ya and T. Shaposhnikova, Theory of Multipliers in Spaces of Differentiable Functions, Pitman, 1985.

[26] S. Müller, T. QI and B.S. YAN, On a new class of elastic deformations not allowing for cavitation, Ann. Inst. Henri Poincaré, 11 (1994), 217-243.

[27] S. Müller and S. Spector, An existence theory for nonlinear elasticity that allows for cavitation, Arch. Rational Mech. Anal., 131, n⿳ 1 (1995), 1-66.

[28] P. Pansu, Difféomorphismes de p-dilatation bornées, Ann. Acad. Sc. Fenn., 223 (1997), 475-506.

[29] P. PANSU, Cohomologie $L^{p}$, espaces homogènes et pincement, preprint, Orsay 1999.

[30] M. REIMAN, Über harmonishe Kapazität und quasikonforme Abbildungen in Raum., Comm. Math. Helv., 44 (1969), 284-307.

[31] Yu. G. Reshetnyak, Space Mappings with Bounded Distortion, Translation of Math. Monographs, 73 (1989).

[32] Yu. G. Reshetnyak, Mappings with bounded distortion as extremals of integrals of Dirichlet type, Siberian Math. Journal, 9 (1968), 652-666.

[33] Yu. G. Reshetnyak, Sobolev classes of functions with values in a metric space, Siberian Math. Journal, 38 (1997), 657-675.

[34] S. Rickman, Quasi-regular Mappings, Springer Ergebnisse der Mathematik, 26 (1993).

[35] S. Rickman, Topics in the Theory of Quasi-regular Mappings, Aspekte der Mathematik, E12 (Kulkarni and Pinkall eds) (1988).

[36] C. J. Titus and G. S. YounG, The extension of interiority, with some applications, Trans. Amer. Math. Soc., 103 (1962), 329-340.

[37] M. Troyanov, Parabolicity of Manifolds, Siberian Adv. Math., 9, no 4 (1999), $1-25$.

[38] S. K. Vodop'Yanov, Taylor Formula and Function Spaces (in Russian), Novosibirsk University (1988).

[39] S. K. Vodop'yanov, Geometric Properties of Function Spaces (in Russian), D. Sc. Thesis, Novosibirsk, Sobolev Institute of Mathematics (1992).

TOME 52 (2002), FASCICULE 6 
[40] S. K. Vodop'YAnov, Monotone Functions and Quaiconformal Mappings on Carnot Groups, Siberian Math. J., 37, n 6 (1996), 1269-1295.

[41] S. K. Vodop'YAnov, Topological and Geometric Properties of Mappings of Sobolev Spaces with Integrable Jacobian I, Siberian Math. J., 41, n ${ }^{\circ} 1$ (2000), $23-48$.

[42] S. K. Vodop'YAnov, Mappings with Bounded Distortion and with Finite Distortion on Carnot Groups, Siberian Math. J., 40, nº 4 (1999), 764-804.

[43] S. VODOP'YANOV and V. GOL'DSHTEIN, Quasi-conformal mapping and spaces of functions with generalized first derivatives, Siberian Math. J., 17, $\mathrm{n}^{\circ} 4$ (1977), $515-531$.

[44] S. Vodop'yanov and A. Ukhlov, Sobolev Spaces and $(P, Q)$-Quasiconformal Mappings of Carnot Groups, Siberian Math. J., 39, nº 4 (1998), 665-682.

[45] V. A. Zorich and V. M. Kesel'man, On the Conformal Type of a Riemannian Manifold, Func. Anal. and Appl., 30 (1996), 106-117.

[46] V. A. Zorich, A theorem of M.A. Lavrent'ev on quasiconformal space maps, Mat.Sb., 74, 116 (1967), 417-433; English transl. in Math. USSR Sb., 3 (1967).

Manuscrit reçu le 7 novembre 2000 , révisé le 26 avril 2002 ,

accepté le 26 juin 2002.

\section{Marc TROYANOV}

E.P.F.L.

Institut de Mathématiques

CH-1015 Lausanne (Switzerland).

marc.troyanov@epfl.ch

Sergei VODOP'YANOV,

Sobolev Institute of Mathematics

Novosibirsk 630090 (Russia).

vodopis@math.nsc.ru 